\title{
Collaborating to Achieve Quality Work in a Minimal Processing Environment
}

The ACRL Rare Books and Manuscripts Section explored collaborative relationships at its 2010 preconference meeting in Philadelphia, with a specific aim of determining circumstances in which working together accomplishes more than working alone, and to suggest creative but practical solutions that can be applied to the challenges that archivists and special collections librarians are confronting. One such example is the Philadelphia Area Consortium of Special Collections Libraries' (PACSCL) "Hidden Collections" Processing Project, a 27-month initiative that began in July 2009. None of what this project has accomplished to date could have been completed without the collaborative efforts made by the entire PACSCL community, the participating repository staff, and the project staff who share their experiences, the lessons they learn, and their advice for future work.

PACSCL was created in 1985 when 16 member repositories began collaborating to promote the historically and intrinsically valuable collections held in their respective institutions. During the last quarter of a century, PACSCL has developed expertise through working together on projects and exhibits, and now counts 35 repositories as members. Recently, PACSCL has undertaken centralized projects that address challenges common across many institutions and provide increased exposure to collections and repositories. The number of repositories in close proximity and the progressive spirit of repository staff have resulted in successful collaborations, both in those based on particular projects and as a community of support and collegiality.

In 2006, PACSCL received a grant from the Council of Library and Information Resources (CLIR) to survey unprocessed, underprocessed, and underdescribed archival collections at 22 area repositories. Christine Di Bella, Archivist and Project Director, led the PACSCL Survey Initiative Project, a 30-month project. Together, she and two collection surveyors, John Armstrong and Jennifer Barr, surveyed over 2,100 archival collections, making most of them available to the public via a collection-level record. This outstanding project resulted in many benefits to the PACSCL community, including "unhiding" some of the extraordinary collections 
in these repositories that were unused or underused by the research community. By assigning a Research Value Rating, a fair and systematic methodology for prioritizing processing emerged that has been adopted by other consortial projects around the country as well as being included in the Archivists' Toolkit Assessment Module.

This survey project led to the current PACSCL/CLIR Hidden Collections Processing Project, for which PACSCL received \$500,000 from CLIR to process and make accessible approximately 200 "hidden" collections held by 23 participating repositories. Using data from the survey, these repositories were encouraged to nominate 10 of their most valuable collections (those receiving a research value of 7 or above out of 10) to be processed and to identify 10 finding aids to be updated and converted to Encoded Archival Description (EAD). At the start of the project, 114 collections were in line for processing and 82 additional finding aids were marked for encoding. In addition, PACSCL sought to provide a centralized finding aid resource for researching the country's political, economic, social, religious, and cultural experiences, as well as Philadelphia's role in shaping America, its cultures, and its citizens. The ambitious goals of the project and the time allotted to complete these goals made it necessary to experiment with large-scale minimal processing for collections ranging from the 17th to 21 st centuries, from which new workflows and strategies should emerge to help repositories deal with the common challenges they face.

Consortial cooperation began with the writing of the project grant and the establishment of several different committees. The PACSCL Oversight Committee deals with major issues related to the overall project; the Standards Committee, which was established before the project began, developed processing manuals and made those decisions that required acceptance by all participating repositories; the Technology Committee assists the project staff as well as repository staff with any project-related computer issues; and the PACSCL Finding Aids Site Committee makes decisions on the centralized finding aid Web site. Repository staff members work closely with project staff to provide subject-based knowledge that increases understanding of a collection and improves the finding aid, the "final product" of the collection processing. In addition, repository staff has helped to identify which aspects of the project do and do not work, and to offer suggestions for improving it.

One of the many challenges with which PACSCL and nearly every archival repository in the country contend are backlogs: the collections on the shelves that are unprocessed and therefore unavailable to researchers. Backlog is generally not indicative of work left undone, but of the efforts repositories have made to save amazing materials from trash cans, basements, attics, and eBay. Backlog exists because processing is a time-intensive and skilled craft and archives have tradition- 


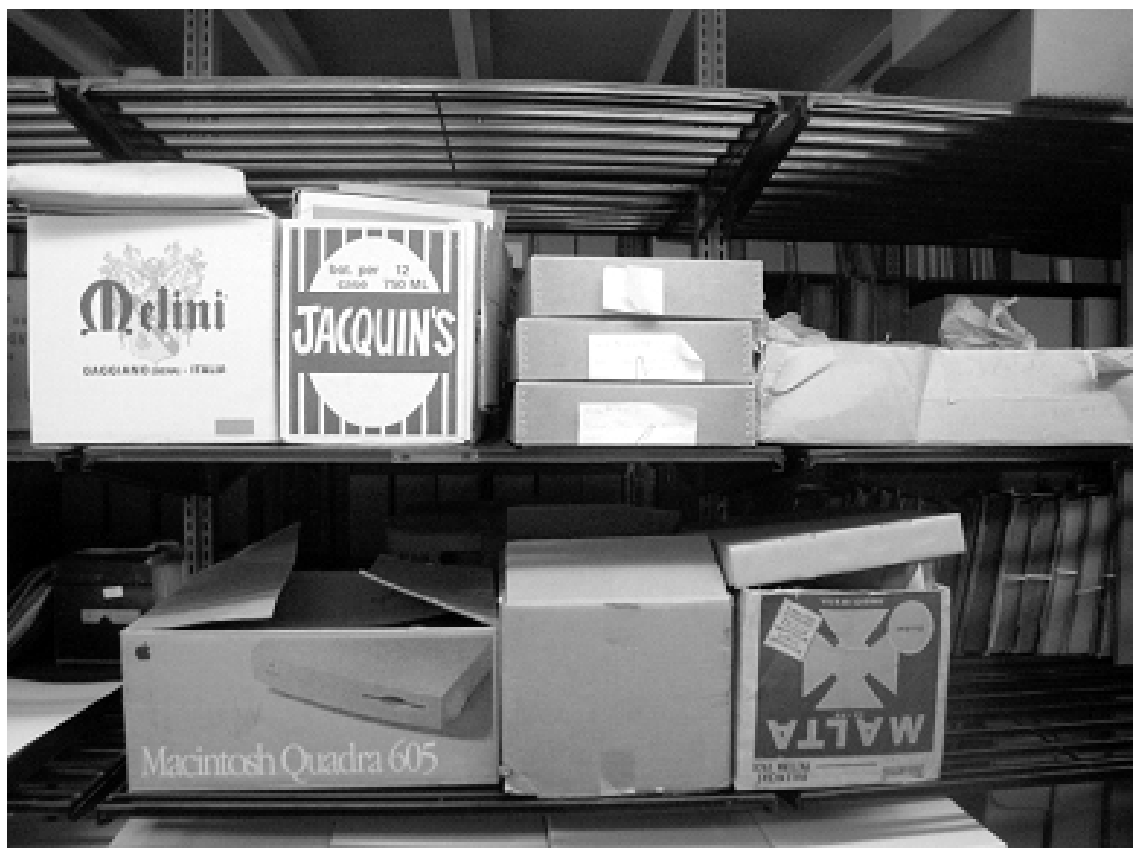

Backlog at one of the PACSCL repositories.

ally been underfunded and understaffed efforts. Backlog is not new, but efforts have recently intensified to increase the speed of backlog reduction. The concept of "minimal processing" was introduced in 2005 by Mark Greene and Dennis Meissner, who recommend that the meticulous efforts characterized by "good" processing be minimized in the case of late 20th-century institutional records to make them accessible to the public at a faster rate. ${ }^{1}$ Despite a storm of controversy, minimal processing has been embraced by many archivists facing decades of work they could not complete if they were to employ more traditional processing methods.

Another challenge that PACSCL and many other repositories face is the lack of standardization with respect to technology, authority control, processing practices and finding aids. Within PACSCL repositories, technological assets are wildly varied, ranging from institutions with dedicated information technology departments, to institutions with contract IT staff who are paid only when problems arise that cannot be solved in-house. Authority control across repositories also becomes a significant problem when developing a shared finding aid repository with faceted searching. In PACSCL's shared EAD repository, each individual institution's "standardized" authorities need to merge with 22 others. Because the ultimate goal is providing a centralized resource for researchers, their search results may not identify all the available resources if authorities do not match in faceted searching.

1. Mark A. Greene and Dennis Meissner, "More Product, Less Process: Revamping Traditional Archival Processing," American Archivist 68, no. 2 (2005): 208-63. 
The project's Standards Committee, consisting of interested PACSCL staff, met well before the project team was in place and determined that all participating repositories would be required to use the Archivists' Toolkit, a collections management database system, and that the project would employ Describing Archives: A Content Standard (DACS). ${ }^{2}$ The Library of Congress and the Art and Architecture Thesaurus would be the sources for obtaining authorized name and subject headings.

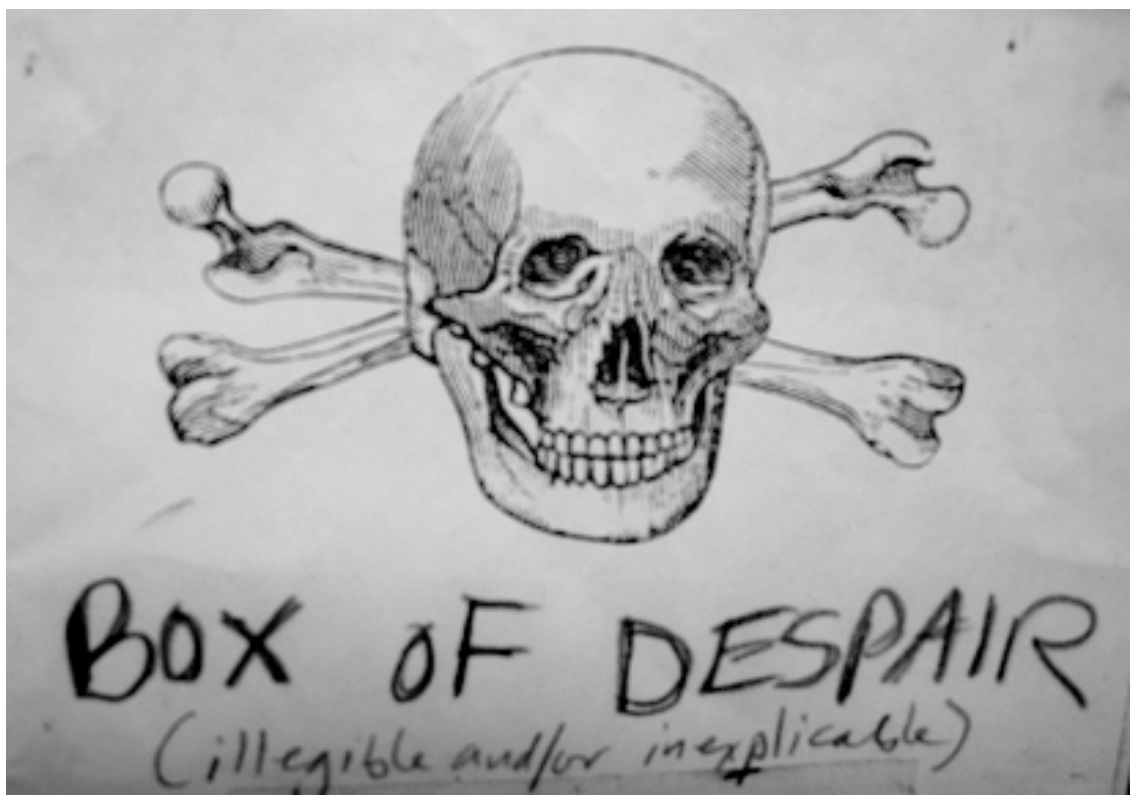

Box of Despair found in the Douglas and Dorothy Steere papers at Haverford College.

Based on the project's available funding and timeline, it was decided that the collections would be minimally processed, even though they did not exclusively consist of "late 20th century institutional records." In fact, many of these collections are manuscript collections from the 18th and 19th centuries. Moreover, Greene and Meissner suggested that minimal processing should be completed in 4 hours per linear foot versus 8 to 15 hours per linear foot, a generally accepted rate for traditional processing. To complete the PACSCL/CLIR Hidden Collections processing project, collections would need to be processed at the rate of 2 hours per linear foot, which proved possible, but at the expense of the project's final product (that is, an adequate description of the collection). Moreover, the CLIR grant required the use of students as processors, who would, in all likelihood, be slower processors than the experienced archivists. Collections that were reasonably well organized could possibly be arranged and described in 2 hours per linear foot; but, as

2. Describing Archives: A Content Standard (Chicago: Society of American Archivists, 2007). 
Courtney Smerz, the project archivist, and I, the project manager, began exploring the collections to be processed, frightening box labels such as "box of despair," "photo chaos," "to be puzzled out," and "absolutely no idea" began surfacing. We therefore determined that a two-step approach would yield the most efficiency and quality of product.

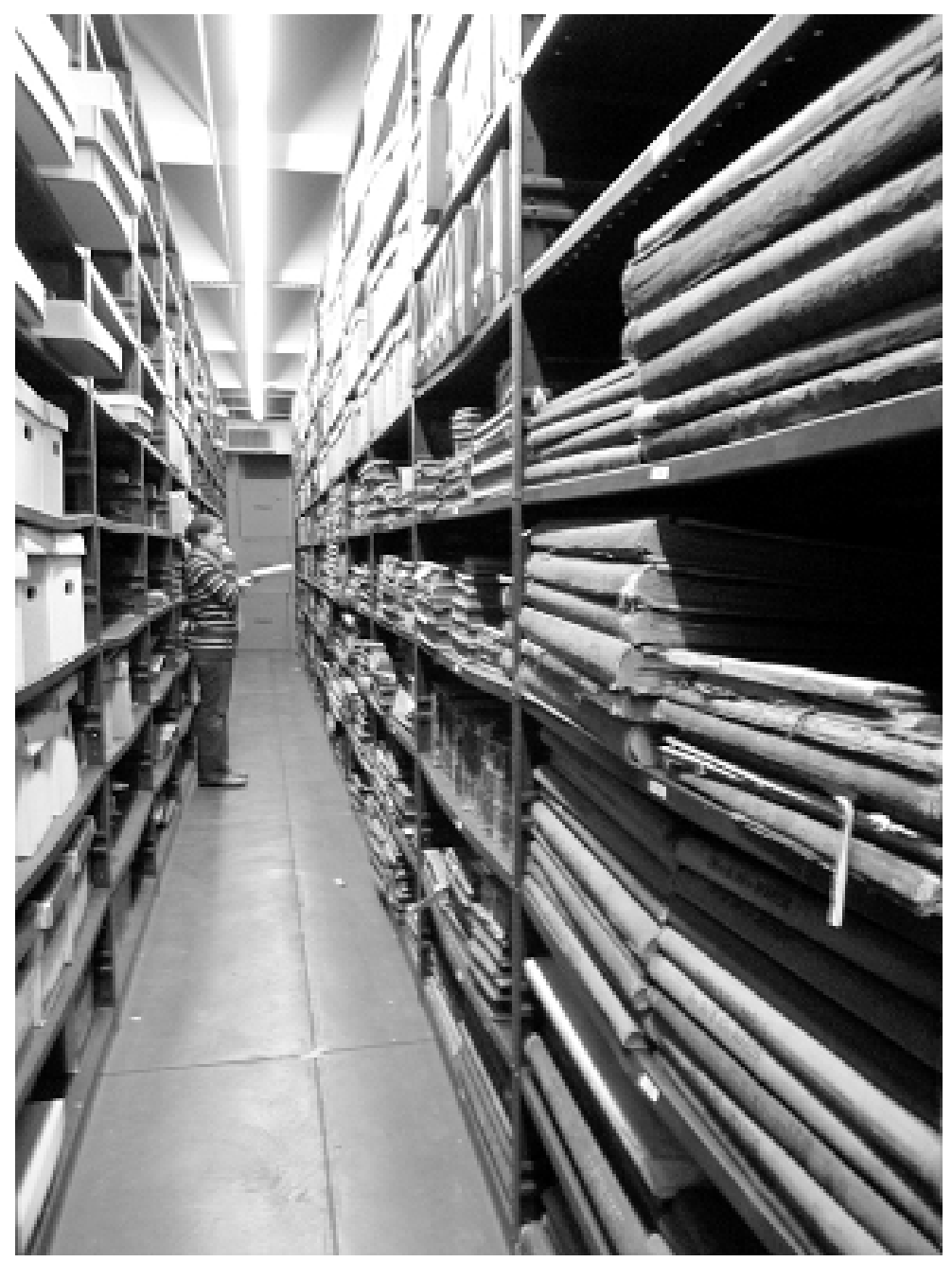

Courtney Smerz, project archivist, creating a processing plan at the Historical Society of Pennsylvania.

The first step occurs before the actual processing begins: we create processing plans for each collection, develop preliminary biographical/historical notes, and propose authority terms, which allows the processors to begin working with a collection immediately. The processors then have the full two hours per linear foot to intellectually and physically arrange the collection, write both an abstract and a scope and content note, and enter the container list into the Archivists' Toolkit. The 
processing plans are just that: proposed plans. They are designed to give student processors the opportunity to make the best possible processing decisions in the shortest amount of time. Processors are encouraged to discuss the proposed series with each other, the repository staff, and project staff to ensure that the collections receive comprehensive treatment within a limited time frame.

It is important to note that " 2 hours per linear foot" refers to "physical processing" and does not take into account the time required for initially looking through the collection, researching and writing the biographical note, proposing series, or identifying potential issues. Such tasks are enormously time intensive yet considered vital to promoting the collections and providing context to their contents, and the time involved in these efforts is currently being tracked separately. Our efforts for processing actually merge both minimal processing and maximal processing, introduced by Rob Cox: ${ }^{3}$ we are minimizing the physical work with a collection while trying not to forfeit description.

To determine the rate of processing for each collection and whether it was a good candidate for minimal processing, we developed worksheets to record processingrelated information. Preservation needs are tracked as well as potential material for digitization and/or exhibits. The worksheets have proved to be very valuable: project staff knows which collections were physically processed in 2 hours per linear foot and which ones took twice that time. Notes supplied by the processors assist other project staff to determine why a collection took twice the time anticipated. As a result, it is clear that age does not determine a collection as a candidate for minimal processing - instead, it is the manner in which the collection was initially organized. An 18th-century collection arranged in volumes or folders can be processed far more quickly than a collection of late 20th-century papers that have been carelessly tossed into a box.

Philadelphia is an ideal city for this type of project. In addition to the dense population of archival repositories, there are also two graduate programs that provide training in archives: Drexel University and Temple University. Student processors are often working toward their MLIS, usually with a concentration in archives, and therefore require no introduction to the basic tenets of archives. Centralized training of staff, in the form of "boot camps," has proven to be one of the most important standardizations implemented. Students are trained in three-day intensive sessions. Day One covers minimal processing, the Archivists' Toolkit, and project requirements for standards and quality. Days Two and Three focus on hands-on

3. Rob Cox, "Maximal Processing, or Archivist on a Pale Horse," Journal of Archival Organization 2, no. 2 (Jan. 2010). Available online at http:/ / umass.academia.edu/RobertCox/Papers/364454/Maximal_processing_or_archivist_on_a_pale_horse. [Accessed 31 January 2011]. 


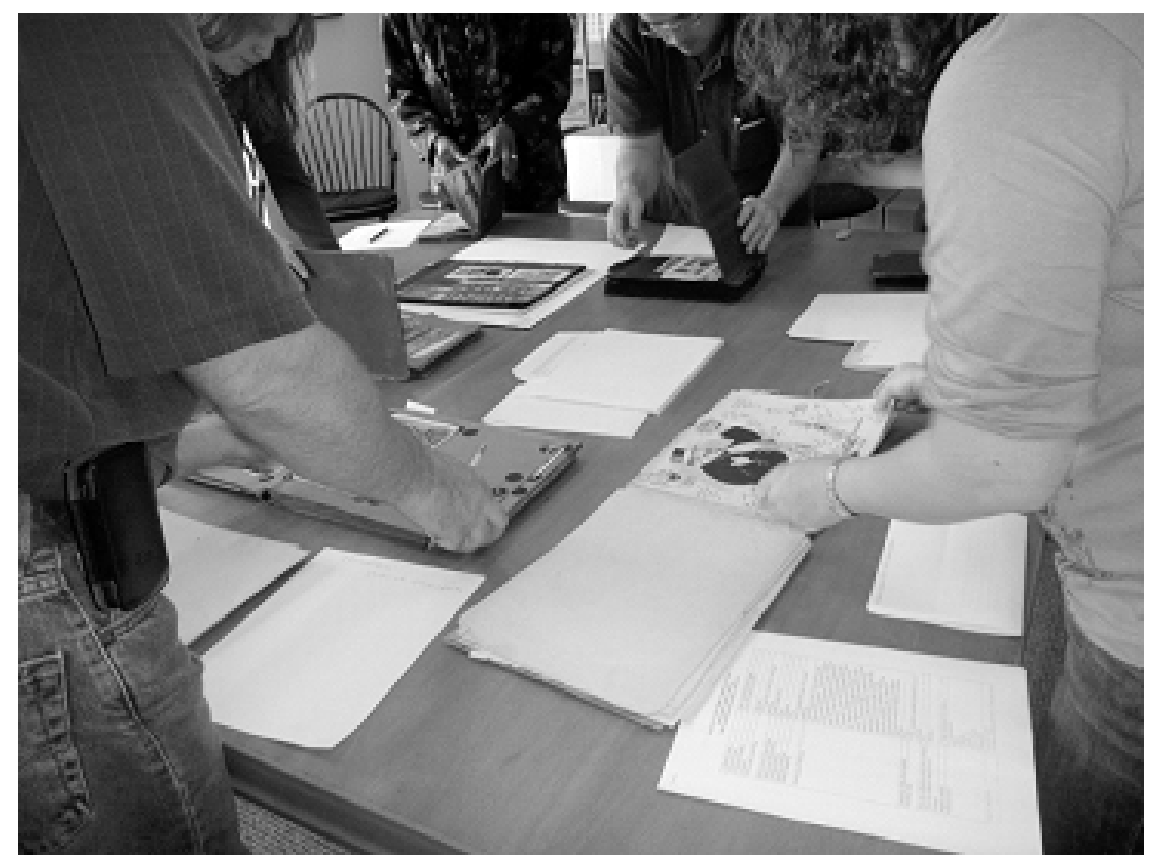

Spring 2010 Boot Camp at the Independence Seaport Museum J. Welles Henderson Archives and Library.

work, minimally processing collections and entering them into the Archivists' Toolkit. The training is not repository specific, so the students are able to work with any of the collections, despite major differences between the repositories. These boot camps have also been used to train other staff, interns, and consultants, both within and outside the PACSCL repositories.

The project staff has been blessed with student processors who are hard working, dedicated, and enthusiastic. The large bulk of the collections have been processed by these students. (Courtney and I process those that clearly cannot be completed within the time frame allotted.) The student processors who have been involved with the project to date include Megan Atkinson, Celia Caust-Ellenbogen, Dan Cavanaugh, Jennifer Duli, Megan Good, Christiana Dobrzynski Grippe, Michael Gubicza, Rebecca Koch, Devin Manzullo-Thomas, Jenna Marrone, Sarah Newhouse, Leslie O’Neill, Laurie Rizzo, Eric Rosenzweig, Brian Stewart, and Forrest Wright. As of April 2011, these passionate current and future archivists have processed 97 collections at 18 different institutions. Another 53 collections have been entered or updated by Garrett Boos, the Archivists' Toolkit Cataloger for the project.

While processing the collections is the students' primary task, they also have actively contributed to the project blog, featuring insights on the project, minimal processing, and a multitude of other processing issues. Their opinions and con- 
cerns have, on many occasions, guided the development of the project and made us aware of issues that require attention.

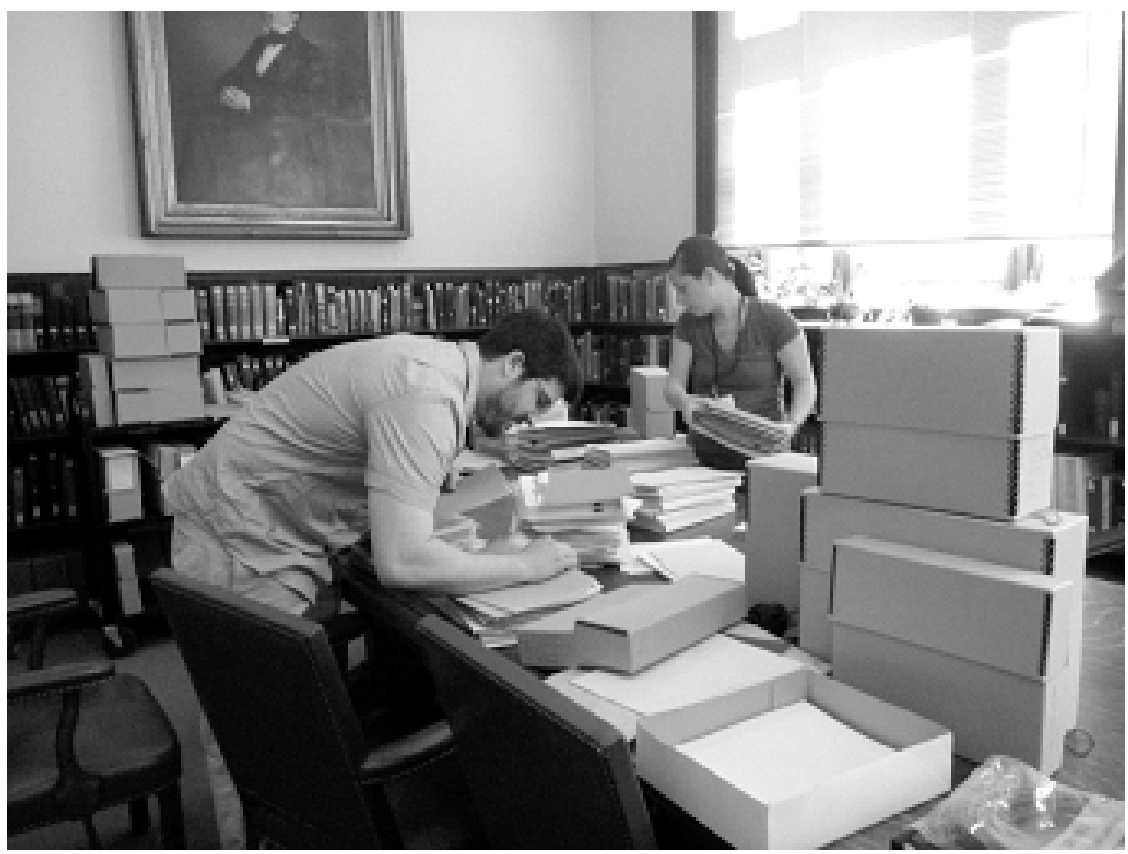

Forrest Wright and Leslie O'Neill processing the College of Physicians of Philadelphia Library records at the College of Physicians of Philadelphia.

In addition to the physical processing that the project has completed, the centralized finding aid site, PACSCL Finding Aids, (http:/ / dla.library.upenn.edu/dla/ $\mathrm{pacscl} / \mathrm{index} . \mathrm{html}$ ) is online and new finding aids are continually being added to it. This site provides an excellent starting place for primary resource research. Users may search for a single topic across all included repositories and discover how widely dispersed related collections are in Philadelphia.

Although the entire project team agrees that 2 hours per linear foot is entirely too fast to process most collections, we have been pleased to discover what can be accomplished with rapid minimal processing when it is applied to the right type of collection. The project's finding aids, for the most part, are fairly thorough, and universally provide access that was not available prior to the project's processing. This project is a first step, not a final product. Every collection processed could benefit from additional work. It is hoped that repository staff will now identify those collections or segments of collections for fuller processing based on their awareness of what is in the collection and the evidence provided by researchers as to what materials are most frequently used. Despite these hopes, we are aware that many of these collections may never receive further work—staffing and budgets, in 
many cases, will not allow for spending money on already processed material. As a result, it is our intention that every finding aid we create be able to stand on its own merits.

One of the project's many goals is to provide guidelines, based upon findings, that could be adapted by other repositories to process their backlogs, a goal we believe we have achieved. We are confident that the practices developed and refined in the PACSCL/CLIR project can readily be adapted by both small and large repositories, which may or may not have significant staff or technological assets. All of the manuals and materials we used and/or developed are available on the project's Web site: http: / clir.pacscl.org. While a centralized finding aid site, as created by the University of Pennsylvania, is an added benefit, repositories without such technical expertise can use the stylesheet provided by the Archivists' Toolkit and can create pdf- or html-formatted finding aids. The Web site also provides documentation of the project itself as transparently as possible, as well as efforts that have been made to improve the efficiency of processing and quality of the finding aids. All processors and repository staff have been encouraged to voice their opinions on the project and to write about the collections they have processed on a project blog, sometimes resulting in researchers and enthusiasts locating the collections before the finding aids have been completed.

It is worth repeating that working at the rate of 2 hours per linear foot is not ideal, and the creation of usable finding aids requires preprocessing work by professional staff. Ideally, we would recommend that the processing time involved should be based on the level of processing appropriate to the collection and the projected use it will receive. On some occasions, item-level description may be necessary for researchers to truly benefit from a collection; more often than not, good descriptive folder titles, or even series- or collection-level processing, will provide researchers with the information they need. On other occasions, a series- or collection-level description will truly be all that a researcher needs to obtain access to the material in a collection. Ultimately, the determination should be made on a collection-bycollection basis that should result in the best treatment for collections and a refinement of methods for prioritizing collections for processing. Recently we provided training to ten Council of Independent Colleges librarians with archival collections in a session funded by the Council on Libraries and Information Resources, and we await, with excitement, feedback on the success of implementing our methodology.

Because providing access to these incredible collections is the ultimate goal of this project, we are making efforts to introduce the project and finding aid sites to communities of users. We have supported workshops in connection with National 
History Day in Philadelphia and are working with teachers who develop lesson plans using primary sources. If one researcher's perspective on history is changed as a result of a collection processed by the project, I consider the project a success.

For the many repositories that are unable to support traditional processing, alternative approaches needed to be identified. The approach we have developed for the PACSCL/CLIR Hidden Collections Processing Project has worked across two dozen vastly different institutions with a wide variety of collections, spanning five centuries and numerous subjects - and it can be done at a rate at least twice as fast as what many repositories are doing. Because we know that our generalized structure and workflow can accommodate numerous variables, we propose that it can not only work in other disparate consortial settings, but it would be even more successful if adopted in more controlled settings, such as single institutions or small consortia with holdings having narrower ranges of subject, formats, timeframes, and collection types. We hope that the lessons we have learned so far continue to be tested in other settings.

SPECIALIZING IN 19TH CENTURY EPHEMERA

- Juvenile Books \& Ephemera

- Historical Memorabilia

- Popular \& Social Culture

- Victoriana

Sheryl Jaeger \& Ralph Gallo

- School girl \& folk art

- Bookseller ephemera Tolland, CT 06084 860.872 .7587

\section{\$heryl Haeger Appraisal Services}

Full range of appraisal \& consulting services including

* $\quad$ IRS appraisals for donations \& estate settlements

* $\quad$ Appraisals for insurance \& estate planning

Special emphasis on Juvenile Books \& Ephemera, Popular Culture, Archives, Paper Americana, Historical Memorabilia and Love \& Friendship, 